\title{
L'ARN satellite du virus de la mosaïque du concombre II. Etude de la relation virus-ARN satellite chez divers hôtes
}

\author{
Mireille JACQUEMOND \& Jean-Paul LEROUX \\ I.N.R.A., Station de Pathologie végétale, Centre de Recherches d'Avignon, F 84140 Montfavet.
}

\section{RÉSUMÉ \\ Virus de la mosaïque du concombre, \\ ARN satellite, \\ Interférence, \\ Réduction de symptômes.}

\begin{abstract}
Le lien entre la présence d'ARN-5 (ARN satellite) dans un inoculum de VMC et le développement de la nécrose létale chez la tomate a conduit à utiliser le «test nécrose» au cours de l'étude des proprićtés biologiques de plusieurs isolats d'ARN satellite.

Une dose de $5 \times 10^{-4} \mu \mathrm{g} / \mathrm{ml}$ (dose minimale) d'ARN-5 est requise pour que sa réplication soit assurée en présence du génome viral. A des concentrations inférieures, sa synthèse devient aléatoire.

Plusieurs souches de VMC, à l'origine dépourvues d'ARN satellite, peuvent assurer la synthèse d'ARN-5 de provenances différentes, même s'il est présent en concentration faible dans l'inoculum. La quantité d'ARN-5 produit est déterminée par la nature du génome viral, par celle de l'ARN-5 et également par la nature de l'espèce hôte utilisée pour la propagation du virus.

Divers hôtes du VMC permettent la synthèse de l'ARN-5 présent à la dose minimale dans l'inoculum, mais avec des efficacités variables selon l'espèce considérée. Les Solanacées sont des hôtes plus propices à la multiplication de l'ARN satellite que les Cucurbitacées.

La présence d'ARN-5 dans un isolat, qui se traduit par une importante diminution de la synthèse des ARN du génome, provoque, chez plusieurs hôtes du virus, une nette réduction des symptômes comparativement à ceux qui sont obtenus après infection par le génome viral seul.
\end{abstract}

\section{SUMMARY}

Cucumber mosaic virus, Satellite RNA, Interference,

Reduction of symptoms.

\section{Cucumber mosaic virus-associated RNA-5 \\ II. Virus-satellite RNA relationships in various host plants}

The correlation between the presence of RNA-5 in a CMV inoculum and the development of lethal necrosis on tomato plants has led to the use of the «necrosis test $»$ for studying some biological properties of several satcllite RNA isolates.

A concentration of $5 \times 10^{-4} \mu \mathrm{g} / \mathrm{ml}$ (minimal dose) of RNA-5 is necessary for optimal replication when added to viral genome. At lower concentrations, its synthesis is unpredectable.

Several CMV strains free of satellite RNA can support the synthesis of RNA-5 isolated from other strains, even if it is present in very low quantity in the inoculum. The amount of satellite RNA recovered is determined by the helper strain, the RNA satellite isolate and the host plant on which the virus is propagated. Various hosts susceptible to CMV allow RNA-5 synthesis from the minimal inoculum, but with different efficiencies according to the species. The capacity to produce RNA-5 is better on Solanaceac than on Cucurbitaceae.

The presence of RNA-5 in an isolate greatly reduces viral RNA replication and provokes, in several plant spccies, a conspicuous reduction of disease symptoms, in comparison with those obtained after infection by the genome alone.

\section{INTRODUCTION}

L'ARN satellite du VMC a fait l'objet de nombreuses études ces dernières années. Les travaux poursuivis aux U.S.A., en Australie, en France et au Japon suggèrent l'existence de 3 types d'ARN satellite selon des critères biologiques et structuraux.

L'ARN-5, ou CARNA-5, a été mis en évidence dans les nucléoprotéines du virus par KAPER et al. (1976). Des études à caractère biochimique ont permis de confirmer sa nature satellite (DIAZ-RUIZ \& KAPER, 1977 ; LOT et al.,
1977) et d'établir pour la souche D, la séquence complète (RICHARDS et al., 1978). Il a par ailleurs été établi que les ARN-5 isolés de différentes souches de virus possèdent des structures primaires très voisines (KAPER \& TOUSIGNANT, 1978 ; RICHARDS et al., 1978). Ils peuvent être répartis en 2 groupes selon le faciès maladif développé par des tomates (Lycopersicon esculentum Mill.) infectées par des inoculums les contenant :

- ARN-5 de type D : son association au génome viral provoque la modification totale de la réponse systémique de 
la tomate qui extériorise un syndrome nécrotique létal caractéristique (KAPER \& WATERWORTH, 1977 ; JACQUEMOND \& LOT, 1981). Les récents travaux de TAKANAMI (1981) suggèrent que l'ARN-5 de la souche qu'il étudic peut être rattaché à ce groupe ;

- ARN-5 de type R: sa présence dans l'inoculum se traduit par la réduction des symptômes de mosaïque et de filiformisme dus à l'infection par le génome viral seul (JACQUEMOND \& LOT, 1981).

En Australie, Gould et al. (1978) ont également décrit l'existence du $5^{\mathrm{c}}$ composant dans l'ARN du VMC et prouvé sa nature satellite. Cet ARN, dénommé SAT-RNA, provoque également, chez la tomate, la réduction des symptômes causés par le virus, mais il ne présente que 70 p. 100 d'homologies de séquence avec l'ARN-5 (Mossop \& FRANCKI, 1979).

Nous présentons ici les résultats obtenus au cours de l'étude de la relation trilatérale virus-ARN satellite-hôte. Ils montrent que les ARN-5 des souches françaises que nous avons considérées possèdent de nombreuses proprićtés communes avec les CARNA-5 et SAT-RNA respectivement étudiés par les équipes américaine et australienne, indépendamment de lcur aptitude nécrogène.

\section{MATÉRIEL ET MÉTHODES}

\section{A. Les souches de virus. Purification des nucléoprotéines et ARN viraux}

Les souches $\mathrm{I}_{17} \mathrm{~F}$ et $\mathrm{I}_{17} \mathrm{~N}$ sont issues du clonage, sur Vigna sinensis (L.) Endl., d'un même isolat naturel du virus provenant d'une tomate atteinte de mosaïque au champ. Elles se différencient essentiellement par la présence d'ARN-5 de type $D$ dans la souche $I_{17} N$ et donc, par lcurs propriétés pathogènes chez la tomate.

Les autres souches expérimentées, les méthodes de purification du virus, de fractionnement des ARN génomiques et de l'ARN-5, d'analyse des composants nuclćiques en gel de polyacrylamide ont été précédemment décrites (JACQUEMOND \& LOT, 1981).

\section{B. Mise en évidence de la synthèse de l'ARN-5}

Le développement de nécrose létale chez la tomate cst utilisé comme révélateur de la présence d'ARN satellite de type $\mathrm{D}$ dans l'inoculum. Chaque test est réalisé sur 10 tomates var. «Monalbo ». Les plantes sont inoculées à un stade juvénile (18 à $20 \mathrm{j}, 2$ à 3 feuilles dévcloppées) par des préparations d'ARN viral purifié, diluć dans une solution de phosphate disodique $0,03 \mathrm{M}, \mathrm{pH} 8,3$, stérile, additionnées de carborandum ( $400 \mathrm{mesh}, 75 \mathrm{mg} / \mathrm{ml}$ ), ou par des extraits bruts de plantes infectées, préparés dans la même solution saline $(4 \mathrm{ml} / \mathrm{g})$ en présence de DIECA $(0,2 \mathrm{p} .100)$ et additionnés de carborandum et de charbon végétal activé ( $75 \mathrm{mg} / \mathrm{ml})$. Durant leur culture et leur incubation, les tomates sont maintenues dans un compartiment de serre dont la température est régulée $\left(16-17^{\circ} \mathrm{C}\right.$ durant la nuit, $23-24^{\circ} \mathrm{C}$ durant lc jour), équipé d'un dispositif de lumière additionnelle afin d'assurer $16 \mathrm{~h}$ de jour toute l'année et protégé vis-à-vis des insectes.

L'ARN-5 de type $\mathrm{R}$ peut être détecté grâce à l'atténuation des symptômes que développent les tomates lorsqu'il se trouve dans l'inoculum. Sa présence est toutefois confirmée par l'analyse, en gel de polyacrylamide, des composants ARN des nucléoprotéines purifiées à partir de 10 tomates infectécs dans les mêmes conditions que précédemment.

\section{RÉSULTATS}

\section{A. Recherche de la dose minimale d'ARN-5 suffisante pour que sa synthèse soit assurée}

Les pourcentages de tomates nécrosées après leur infection par des inoculums constitués des ARN de la souche $\mathrm{I}_{17} \mathrm{~F}$ - inducteurs de filiformisme - additionnés de doses croissantes d'ARN-5 de type D (souche $\mathrm{I}_{17} \mathrm{~N}$ ) figurent dans le tableau 1.

La dose de $5 \times 10^{-4} \mu \mathrm{g} / \mathrm{ml}$ d'ARN-5 est suffisante pour que la quasi-totalité $(97,1$ p. 100$)$ des plantes inoculécs extériorisent le syndrome nécrotique. L'analyse du contenu nucléique des particules purifiées à partir d'un de ces lots de tomates révèle que l'ARN satellite représente alors 45 p. 100 environ de l'ARN total encapsidé (tabl. 4).

Quand la concentration de l'ARN-5 est inférieure à $5 \times 10^{-4} \mu \mathrm{g} / \mathrm{ml}$, le pourcentage de tomates qui se nécrosent diminue rapidement avec la dose, suggérant que la synthèse de l'ARN satellite est alors alćatoire.

\section{TABLEAU 1}

Proportion de tomates nécrosées en fonction de la concentration de l'AR.N-5 de type $D$ dans l'inoculum

Proportion of tomato plants displaying necrosis according to the concentration of RNA-5 - D Type - in the inoculum

\begin{tabular}{ccc}
$\begin{array}{c}\text { Concentration de l'ARN-5 } \\
\text { dans linoculum }\end{array}$ & $\begin{array}{c}\text { Nombre de plantes } \\
\text { éprouvées }^{\mathrm{c}}\end{array}$ & $\begin{array}{c}\text { \% de plantes } \\
\text { nécrosécs }^{\mathrm{d}}\end{array}$ \\
\hline 0 (témoin) & 210 & 0 \\
$5 \times 10^{-4}$ & 40 & 2,5 \\
$5 \times 10^{-}$ & 50 & 4,0 \\
$5 \times 10^{-7}$ & 110 & 8,2 \\
$5 \times 10^{\circ}$ & 100 & 16,0 \\
$5 \times 10^{-5}$ & 140 & 67,9 \\
$5 \times 10^{-}$ & 140 & 97,1 \\
$5 \times 10^{-3}$ & 60 & 100 \\
$5 \times 10^{-2}$ & 30 & 100
\end{tabular}

"ARN-5 de la souche $I_{17} \mathrm{~N}$, en $\mu \mathrm{g} / \mathrm{ml}\left(\mathrm{I}_{17} \mathrm{~N}\right.$ RNA-5, in $\left.\mu \mathrm{g} / \mathrm{ml}\right)$.

b. ARN de la souche $I_{17} \mathrm{~F}$ apporté à la dose de $20 \mu \mathrm{g} / \mathrm{ml}$ (Inocula contained $20 \mu \mathrm{g} / \mathrm{ml} \mathrm{I} \mathrm{I}_{17} \mathrm{~F}$ RNA).

Plusieurs essais (10 tomates par dilution) ont été réalisés tout au long de lannćc : chaque pourcentage a été établi à partir de l'ensemble de ces résultats (Scveral experiments (10 plants per dilution) throughout the year: each valuc results based on overall).

“Dans tous les cas, les plantes non atteintes de nćcrose présentaient les symptômes de filiformisme caractéristiques de la synthèse du seul génome viral (Non necrosed tomato plants showed the mosaic and fern leaf symptoms characteristic of the synthesis of the genome alone).

Une étude comparable a été réalisée avec l'ARN satellite de la souche $\mathrm{R}$ en évaluant sa proportion dans les nucléoprotéines purifićes. Lorsqu'il est apporté à une dose égale ou supérieure à $5 \times 10^{-4} \mu \mathrm{g} / \mathrm{ml}$, l'ARN-5 est synthétisé avec le maximum d'efficacité et se trouve en quantités relatives élevées dans les particules (tabl. 2). La comparaison des rendements en nucléoprotéines montre que ceux-ci sont d'autant plus faibles que le taux de synthèse de l'ARN5 est élevé.

La concentration seuil de $5 \times 10^{-4} \mu \mathrm{g} / \mathrm{ml}$ est définic comme «la dose minimale " d'ARN satellite requise pour que son taux de synthèse, en présence du génome viral, soit optimal. Cette dose seuil n'est pas modifiée lorsque lon augmente ou diminue la concentration des ARN du génome (résultats non présentés). 
TABLEAU 2

Effet de la dose d'ARN-5 de type $R$ dans l'inoculum sur la concentration des particules virales et la proportion relative d'ARN-5 synthétisé chez la tomate

Influence of satellite $R N A-R$ type - concentration on virus yields and relative proportion of $R N A-5$ recovered from tomato plants

Exp. 1

Concentration de l'ARN-5

$(\mu \mathrm{g} / \mathrm{ml})$ dans l'inoculum ${ }^{\mathrm{a}}$

$$
\text { rendement }{ }^{b}
$$
virus $(\mathrm{mg} / \mathrm{kg})$
Exp. 2

rendement virus $(\mathrm{mg} / \mathrm{kg})$

)

$\%$ ARN-5
1972,3

1776,0

1855,0

1507,5

850,5

562,5

372,5

286,1

328,3

287,2

217,8

$5 \times 10^{-5}$

$5 \times 10^{-4}$

$5 \times 10^{2}$

$5 \times 10^{-1}$

126,9

145,6

174,7

47,3

51,2

47,6

2

0
0
0
1,3
6,0
20,0
43,0
47,0
49,8
48,6
52,3

"ARN de la souche $I_{17} F, 20 \mu \mathrm{g} / \mathrm{ml}$ (Inocula contained $20 \mu \mathrm{g} / \mathrm{ml} \mathrm{I} \mathrm{I}_{17} \mathrm{~F}$ RNA).

" Chaque valcur correspond à la purification de 10 plantes infectées (Each yield is given for 10 inoculated plants).

- La proportion relative du satellite est déterminée par la mesure du poids de l'aire découpée de l'électrophorégramme (gel de polyacrylamide $2,4 \mathrm{p} .100$ ) (The relative proportion of RNA-5 was determined by measuring its area by planimetry after electrophoresis on 2,4 p. 100 polyacrylamide gel).

\section{TABLEAU 3}

Aptitude nécrogène d'inoculums correspondant aux combinaisons homologues ou hétérologues de plusieurs souches de virus et d'ARN satellite

Aptitude to induce necrosis of inocula corresponding to the homologous and heterologous combinations of several strains of virus and satellite RNA

\begin{tabular}{|c|c|c|c|c|}
\hline \multirow{2}{*}{$\begin{array}{l}\text { Souche } \\
\text { d'origine } \\
\text { de l'ARN-5 }\end{array}$} & \multirow{2}{*}{$\begin{array}{c}\text { Concentration } \\
(\mu \mathrm{g} / \mathrm{ml}) \\
\text { de l'ARN-5 } \\
\text { dans } \\
\text { l'inoculum }\end{array}$} & \multicolumn{3}{|c|}{$\begin{array}{l}\text { \% de nécrose }{ }^{\mathrm{a}} \text { chez les tomates } \\
\text { inoculées par les différentes } \\
\text { combinaisons utilisant les souches }\end{array}$} \\
\hline & & $\mathrm{I}_{17} \mathrm{~F}$ & K & M \\
\hline \multirow[t]{5}{*}{$1_{1} \cdot \mathrm{N}$} & 0 (Témoin) & 0 & 0 & 0 \\
\hline & $5 \times 10^{-7}$ & 30 & 0 & 0 \\
\hline & $5 \times 10^{-6}$ & 60 & 20 & 20 \\
\hline & $5 \times 10^{-5}$ & 90 & 40 & 90 \\
\hline & $5 \times 10^{-t}$ & 100 & 100 & 100 \\
\hline \multirow[t]{5}{*}{ TL } & 0 (Témoin) & 0 & 0 & 0 \\
\hline & $5 \times 10^{-7}$ & 30 & 0 & 0 \\
\hline & $5 \times 10^{6}$ & 90 & 0 & 10 \\
\hline & $5 \times 10^{-5}$ & 100 & 50 & 50 \\
\hline & $5 \times 10^{4}$ & 100 & 90 & 100 \\
\hline \multirow[t]{5}{*}{$S$} & 0 (Témoin) & 0 & 0 & 0 \\
\hline & $5 \times 10^{7}$ & 10 & 30 & 10 \\
\hline & $5 \times 10^{6}$ & 40 & 20 & 30 \\
\hline & $5 \times 10^{-5}$ & 100 & 40 & 100 \\
\hline & $5 \times 10^{-4}$ & 100 & 100 & 100 \\
\hline \multirow[t]{5}{*}{ D } & 0 (Témoin) & 0 & 0 & 0 \\
\hline & $5 \times 10^{-7}$ & 10 & 0 & 0 \\
\hline & $5 \times 10^{-6}$ & 30 & 20 & 10 \\
\hline & $5 \times 10^{-5}$ & 100 & 40 & 40 \\
\hline & $5 \times 10^{4}$ & 90 & 90 & 90 \\
\hline
\end{tabular}

\footnotetext{
i Toutes les plantes non nécrosées ont développé les symptômes de mosaïque et filiformisme (All non necrotic tomato plants have developed mosaic and fern leaf symptoms).
}

\section{B. Aptitude de plusieurs génomes viraux à assurer la synthèse d'ARN-5 de provenances différentes}

L'étude de la spécificité de la relation virus-ARN satellite a été réalisée en infectant de jeunes tomates avec des inoculums hétérologues, constitués par l'addition d'ARN-5 de type $\mathrm{D}$ isolés de 4 souches différentes $\left(\mathrm{I}_{17} \mathrm{~N}, \mathrm{TL}, \mathrm{S}\right.$ et $\left.\mathrm{D}\right)$ à 3 souches dépourvues d'ARN satellite $\left(\mathrm{I}_{17} \mathrm{~F}, \mathrm{~K}\right.$ et $\left.\mathrm{M}\right)$ et inductrices de filiformisme. Les symptômes obtenus avec ces inoculums montrent que la synthèse des divers ARN-5 est assurée par chaque génome viral, puisque la nécrose se développe quelle que soit la combinaison effectuée (tabl. 3). Comme pour l'ARN-5 $\mathrm{I}_{17} \mathrm{~N}$, la dose minimale des ARN-5 TL ou $S$ nécessaire pour assurer $100 \mathrm{p} .100 \mathrm{de}$ nécrose est de $5 \times 10^{-4} \mu \mathrm{g} / \mathrm{ml}$. L'ARN-5 D semble être moins efficient à cette concentration.

L'analyse du contenu nucléique des particules synthétisées chez des tomates contaminćes par les souches $\mathrm{I}_{17} \mathrm{~F}$ ou $\mathrm{K}$ additionnées de l'un ou l'autre des 3 ARN satellites à la dose minimale, confirme cette possibilité pour le virus assistant d'assurer la synthèse de l'ARN satellite hétérologue (tabl. 4).

La nature de l'ARN-5, comme celle de la souche de virus, intervient au niveau de la régulation de sa propre synthèse. Celle-ci est en effet plus active lorsque le génome utilisé est celui de la souche $\mathrm{I}_{17} \mathrm{~F}$. Dans ce cas notamment, les ARN satellites $S$ et $D$ sont répliqués avec plus d'efficacité que lorsqu'ils sont associés à leur propre génome viral (tabl. 4).

Mais il apparaît également que l'ARN satellite D, est produit en quantité plus faible que les 2 autres ARN-5 lorsqu'ils sont assistés par les souches $\mathrm{I}_{17} \mathrm{~F}$ ou $\mathrm{K}$. Par contre, la réduction de la synthèse virale - conséquence de la multiplication de l'ARN-5 - n'est pas proportionnelle au taux de synthèse de l'ARN-5. En effet, la présence de l'ARN satellite $I_{17} \mathrm{~N}$ a pour effet une diminution plus importante de la quantité de nucléoprotéines que la présence de l'ARN satellite $S$ et sa proportion relative dans les particules virales est plus faible. 


\section{TABLEAU 4}

Quantité de virus synthétisé chez la tomate, en présence et absence d'ARN satellite homologue ou hétérologue, et proportion relative d'ARN satellite dans les nucléoprotéines

Yields of CMV recovered from tomato in the presence and absence from homologous or heterologous satellite $R N A$ and relative proportion of satellite $R N A$ in the nucleoproteins

\begin{tabular}{|c|c|c|}
\hline Inoculumi & $\begin{array}{l}\text { Rendement en virus }{ }^{b} \\
(\mathrm{mg} / \mathrm{kg})\end{array}$ & $\%$ ARN-5 \\
\hline $\mathrm{I}_{17} \mathrm{~F}$ & 1552,0 & 0 \\
\hline $\mathrm{I}_{17} \mathrm{~F}+\mathrm{ARN}-5 \mathrm{I}_{17} \mathrm{~N}$ & 316,0 & 44,7 \\
\hline $\mathrm{I}_{17} \mathrm{~F}+\mathrm{ARN}-5 \mathrm{~S}$ & 531,0 & 49,7 \\
\hline $\mathrm{I}_{17} \mathrm{~F}+\mathrm{ARN}-5 \mathrm{D}$ & 520,0 & 40,3 \\
\hline $\mathrm{K}$ & 432,0 & 0 \\
\hline $\mathrm{K}+\mathrm{ARN}-5 \mathrm{I}_{17} \mathrm{~N}$ & 60,0 & 17,8 \\
\hline $\mathrm{K}+\mathrm{ARN}-5 \mathrm{~S}$ & 84,0 & 27,0 \\
\hline $\mathrm{K}+\mathrm{ARN}-5 \mathrm{D}$ & 117,1 & 6,5 \\
\hline $\mathrm{S}$ & 199,0 & 27,1 \\
\hline $\mathrm{D}$ & 620,0 & 15,4 \\
\hline
\end{tabular}

"Les souches de virus assistant $I_{17} F$ et $K$ ont été apportées sous forme d'ARN aux doses respectives de $20 \mu \mathrm{g} / \mathrm{ml}$ et $40 \mu \mathrm{g} / \mathrm{ml}$. Les ARN-5 ont été additionnćs à la dose de $5 \times 10^{-4} \mu \mathrm{g} / \mathrm{ml}$. Les ARN viraux $\mathrm{S}$ et $\mathrm{D}$, contenant l'ARN-5, ont été inoculés à la concentration de $40 \mu \mathrm{g} / \mathrm{ml}$ (Inocula contained $20 \mu \mathrm{g} / \mathrm{ml}$ and $40 \mu \mathrm{g} / \mathrm{ml}$ helper RNA $I_{17} F$ and $K$ respectively, with $5 \times 10^{-4} \mu \mathrm{g} / \mathrm{ml}$ or without satellite RNA. S and D RNAs, containing RNA-5, were inoculated at $40 \mu \mathrm{g} / \mathrm{ml}$ ).

b Chaque rendement a été établi après la purification de 10 tomates (Yields of virus recovered after purification of 10 tomato plants).

c Proportions déterminées après analyse, en gel de polyacrylamide 2,4 p. 100, des préparations d'ARN purifiées (Proportion of RNA-5 was determincd after electrophoresis of isolated RNAs from each virus preparation in 2,4 p. 100 polyacrylamide gels).

\section{Aptitude de divers hôtes du VMC à assurer la synthèse de l'ARN-5 en présence du génome viral}

KAPER et al. (1976) puis KAPER \& TOUSIGNANT (1977) ont montré que la synthèse d'ARN-5 peut-être dépendante de l'hôte - courgette ou tabac - infecté. Il était intéressant de comparer l'aptitude de divers hôtes du VMC, appartenant à des familles botaniques différentes, à assurer la synthèse de l'ARN satellite. L'étude a été réalisée en infectant plusieurs espèces avec un inoculum riche en ARN-5 nécrogène (souche $I_{17} N$ ) ou, au contraire, n'en contenant que de faibles doses (souche $\mathrm{I}_{17} \mathrm{~F}$ additionnée de $5 \times 10^{-4} \mu \mathrm{g} / \mathrm{ml}$ ou $5 \times 10^{-6} \mu \mathrm{g} / \mathrm{ml}$ d'ARN-5 I $17 \mathrm{~N}$ ). L'efficacité de la synthèse de l'ARN-5 chez ces hôtes a été appréciée soit par la détermination de la quantité de particules produites et de leur contenu nucléique, soit par l'aptitude nécrogène des extraits bruts des plantes ainsi contaminées sur des lots de tomates.

\section{Quantités de nucléoprotéines et d'ARN-5 synthétisés chez} divers hôtes du virus

Les rendements en virus obtenus chez les espèces infectées avec les 2 types d'inoculums ainsi que la proportion de l'ARN satellite dans les nucléoprotéines sont présentés dans le tableau 5.

Chez toutes les espèces, la réduction de la synthèse virale est importante lorsque l'inoculum contient l'ARN-5 en forte concentration. Dans ce cas, la proportion d'ARN-5 est beaucoup plus élevée chez les 4 solanacées éprouvées, chez l'épinard et d'autres hôtes tels que le céleri ou le navet (38 à 45 p. 100 de l'ARN total), que chez les 2 cucurbitacées ou le maïs. Ces solanacées et l'épinard présentent une aptitude particulière à assurer cette synthèse, puisqu'ils permettent

\section{TABLEAU 5}

Quantité de virus synthétisé chez divers hôtes et proportion relative d'ARN-5 dans les nucléoprotéines Yields of virus synthesized on various hosts and relative proportion of RNA-5 in the nucleoproteins

\begin{tabular}{|c|c|c|c|c|c|}
\hline \multirow{2}{*}{ Hôte } & \multirow{2}{*}{$\begin{array}{l}\text { Durée } \\
\text { d'incuba- } \\
\text { tion } \\
\text { (jours) }\end{array}$} & \multicolumn{2}{|c|}{$\begin{array}{l}\text { Rendement en virus } \\
\text { après infection par } \\
\text { les inoculums }\end{array}$} & \multicolumn{2}{|c|}{$\begin{array}{l}\% \mathrm{ARN}-5^{\circ} \\
\text { après infection par } \\
\text { les inoculums }\end{array}$} \\
\hline & & $\mathrm{I}_{17} \mathrm{~N}$ & $\begin{array}{c}\mathrm{I}_{17} \mathrm{~F}+ \\
\text { trace } \mathrm{ARN}-5\end{array}$ & $\mathrm{I}_{37} \mathrm{~N}$ & $\begin{array}{c}\mathrm{l}_{17} \mathrm{~F}+ \\
\text { trace } A R N-5\end{array}$ \\
\hline Lycopersicon esculentum Mill. cv. « Monalbo" & 15 & 249,5 & $\begin{array}{c}1907,0^{d} \\
466,1\end{array}$ & 42,1 & $\begin{array}{c}0 \\
10,4\end{array}$ \\
\hline Nicotiana tabacum L. cv. «Xanthi nc.» & 7 & 438,5 & 758,6 & 45,5 & 19,9 \\
\hline Solanum melongena L. cv. "Violette de Barbentanc" & 8 & 182,9 & 518,8 & 37,6 & 10,4 \\
\hline Capsicum annuum L. cv. "Yolo Wonder" & 8 & 384,3 & - & 42,8 & - \\
\hline Spinacia oleracea L. cv. « Vicking" & 8 & 313,7 & 1347,8 & 41,8 & 7,5 \\
\hline Ocimum basilicum L. & 8 & 16,1 & 28,6 & $+c$ & 0 \\
\hline Apium graveolens L. cv. "Doré Barbicr" & 12 & 11,0 & 742,0 & 38,1 & 0 \\
\hline Brassica napus (L.) Metz. cv. "Blanc dur d'hiver" & 8 & 18,4 & 142,9 & 38,4 & 0 \\
\hline Raphanus sativus L. cv. "Radis de 18 jours» & 8 & 9,5 & 29,7 & $+c$ & 0 \\
\hline Cucumis melo L. cv. "Cantaloup Charentais" & 7 & 219,2 & 559,0 & 14,6 & 0 \\
\hline Cucumis sativus L. cv. «Vert Long Maraîcher» & 7 & 159,5 & 550,0 & 19,0 & 0 \\
\hline Zea mays L. cv. «Golden Cross Bantam» & 12 & 19,8 & 86,6 & 13,7 & 0 \\
\hline
\end{tabular}

"Les rendements sont exprimés en $\mathrm{mg} / \mathrm{kg}$ de feuilles fraîches des divers hôtes soumis à purification (Yields of virus recovered from various hosts are expressed as $\mathrm{mg}$ of nuclcoproteins per $\mathrm{kg}$ of leaf material).

" Les ARN $I_{17} F$ et $I_{17} \mathrm{~N}$ ont été inoculés aux doses respectives de $20 \mu \mathrm{g} / \mathrm{ml}$ et $40 \mu \mathrm{g} / \mathrm{ml}$, l'ARN $\mathrm{I}_{17} \mathrm{~N}$ contenant 45 p. 100 environ d'ARN-5; l'ARN-5 de la souche $I_{17} \mathrm{~N}$ a été ajouté à la souche $\mathrm{I}_{17} \mathrm{~F}$ à la dose de $5 \times 10^{\mathrm{t}} \mu \mathrm{g} / \mathrm{ml}$ ( $\mathrm{I}_{17} \mathrm{~F}$ and $\mathrm{I}_{17} \mathrm{~N}$ RNAs were inoculated at $20 \mu \mathrm{g} / \mathrm{ml}$ and $40 \mu \mathrm{g} / \mathrm{ml}$ respectively, $I_{17} \mathrm{~N}$ RNA containing about $45 \%$ of RNA-5; I 17 N RNA-5 added at $5 \times 10^{-6} \mu \mathrm{g} / \mathrm{ml}$ to $\mathrm{I}_{17} \mathrm{~F}$ RNA).

Proportions déterminćes après analyse, en gel de polyacrylamide 2,4 p. 100, des préparations d'ARN purifiées (Proportion of RNA-5 determined after electrophoresis of isolated RNAs from each virus preparation in $2,4 \%$ polyacrylamide gels)

"La synthèse de l'ARN-5 apporté à la dose de $5 \times 10^{-\hbar} \mu \mathrm{g} / \mathrm{ml}$ dans l'inoculum n'est pas toujours assurće chez la tomate (tabl. 1) (When added at $5 \times 10^{-1 h} \mu \mathrm{g} / \mathrm{ml}$ in the inoculum, RNA-5 is not always replicated in tomato plants (tabl. 1)).

'La proportion d'ARN-5 dans ces préparations n'a pu être déterminćc du fait de la présence d'ARN dégradé migrant à l'extrémité du ge (In two preparations, the proportion of RNA-5 has not becn determined because of degraded RNAs migrating near the end of the gel). 
TABLEAU 6

Aptitude nécrogène des extraits de divers hôtes infectés en fonction de la concentration d'ARN-5 dans l'inoculum initial Necrosis inducing capacity of crude extracts of various infected hosts according to RNA-5 concentration in the inoculum

\begin{tabular}{|c|c|c|c|c|c|}
\hline \multirow{3}{*}{ Hôtc } & \multicolumn{5}{|c|}{$\begin{array}{l}\text { p. } 100 \text { de nécrose }{ }^{\mathrm{a}} \text { chez des tomates rétroinoculées }{ }^{\mathrm{b}} \text { par les } \\
\text { extraits des divers hôtes infectés par les inoculums }\end{array}$} \\
\hline & \multicolumn{2}{|c|}{$\mathrm{I}_{17} \mathrm{~F}+$} & \multicolumn{2}{|c|}{$\mathbf{I}_{17} \mathrm{~F}+$} & \multirow[t]{2}{*}{$\mathrm{I}_{17} \mathrm{~F}$} \\
\hline & ARN-5 & $5 \times 10^{-+} \mu \mathrm{g} / \mathrm{ml}$ & ARN-5 & $5 \times 10^{-6} \mu \mathrm{g} / \mathrm{ml}$ & \\
\hline L. esculentum & 100 & $(2)^{d}$ & 28,0 & $(4)^{d}$ & $0^{\mathrm{c}}$ \\
\hline N. tabacum & 100 & (2) & 86,0 & (5) & 0 \\
\hline S. melongena & 100 & (2) & 32,0 & $(5)$ & 0 \\
\hline C. annuum & 100 & (1) & 40,0 & (4) & 0 \\
\hline$S$. oleracea & 100 & (2) & 100,0 & (4) & \\
\hline O. basilicum & 100 & (1) & 50,0 & (4) & 0 \\
\hline A. graveolens & 100 & (1) & 10,0 & (5) & 0 \\
\hline B. napus & 100 & (2) & 18,0 & (5) & 0 \\
\hline$R$. sativus & 100 & (1) & 25,9 & (3) & 0 \\
\hline C. melo & 100 & (2) & 0 & (5) & 0 \\
\hline C. sativus & 100 & (2) & 2,0 & (5) & 0 \\
\hline Z. mays & 100 & (2) & 0 & (4) & 0 \\
\hline
\end{tabular}

"Les plantes non atteintes de nécrose ont extériorisé les symptômes de mosaïque et filiformisme (Non necrotic tomato plants showed mosaic and fern-leaf symptoms).

"Les rétroinoculations ont été effectuées $10 \mathrm{j}$ après l'infection des divers hôtes (Back inoculations have been done 10 days after infection of the various hosts).

"La souche $I_{17} F$ a été apportée sous forme d'ARN purifié à la dose de $20 \mu \mathrm{g} / \mathrm{ml}$; l'ARN-5 a été purifić à partir de la souche $I_{17} N$ (Inocula contained $20 \mu \mathrm{g} / \mathrm{ml} \mathrm{I}{ }_{17} \mathrm{~F}$ RNA; RNA-5 was purified from $\mathrm{I}_{17} \mathrm{~N}$ strain).

"Nombre d'essais réalisés (Number of experiments).

Tous les hôtes ont été éprouvés plusicurs fois, simultanément ou non (All hosts were tested many times, simultancously or not).

la mise en évidence de 10 à 20 p. 100 d'ARN-5, présent pourtant à l'état de traces dans l'inoculum.

\section{Aptitude nécrogène des extraits des divers hôtes infectés}

La réponse spécifique de la tomate à l'infection par du VMC contenant de très faibles quantités d'ARN satellite non détectables par les méthodes classiques d'analyse des ARN - nous a conduits à utiliser le test nécrose pour mettre en évidence l'aptitude nécrogène d'extraits infectieux des différents hôtes éprouvés. Pour cela, les diverses espèces ont été infectées par l'ARN de la souche $\mathrm{I}_{17} \mathrm{~F}$ additionné d'ARN-5 $\mathrm{I}_{17} \mathrm{~N}$ à la dose minimale $\left(5 \times 10^{-4} \mu \mathrm{g} / \mathrm{ml}\right)$ ou à la dose de $5 \times 10^{-6} \mu \mathrm{g} / \mathrm{ml}$.

Les résultats présentés dans le tableau 6 confirment l'aptitude particulière de certaines espèces à micux assurer la synthèse de l'ARN satellite nécrogène. Ces différences ne sont toutefois révélées que lorsque l'ARN-5 est à l'état de traces dans l'inoculum. En effet, tous les extraits de plantes infectées au moyen de l'inoculum contenant l'ARN-5 à la dose scuil provoquent la nécrose de la totalité des tomates rétroinoculées. La proportion de tomates qui se nécrosent est, par contre, variable sclon l'hôte lorsquc l'inoculum contient $5 \times 10^{-6} \mu \mathrm{g} / \mathrm{ml}$ d'ARN-5. Dans ce cas, les 2 cucurbitacées et le maîs assurent rarement la synthèse de l'ARN satellite.

Le fait que les tomates inoculćes avec les extraits bruts de tous les hôtes infectés par la souche $\mathrm{I}_{17} \mathrm{~F}$ ne présentent que des symptômes de filiformisme (tabl. 6) confirme l'absence d'ARN-5 dans cet isolat.

\section{Influence de la synthèse de l'ARN-5 sur l'expression des symptômes chez plusieurs hôtes}

Chez la tomate, l'inoculation de plusicurs souches de virus assistant additionné d'ARN satellite de diverses provenances, provoque généralement l'apparition du syndrome nécrotique. L'ARN satellite de la souche $\mathrm{R}$ est en effet le seul de nos isolats qui, associé au VMC, conduise à une atténuation importante des symptômes de mosaïque et de filiformisme. La démonstration de l'effet de la synthèse de l'ARN satellite de type D dans l'expression d'un syndrome nouveau chez la tomate, nous a amenés à suivre avec attention l'évolution des symptômes provoqués par le VMC, en présence ou absence d'ARN satellite, chez diverses espèces.

Plusieurs hôtes, appartenant comme la tomate à la famille des Solanacées ou à d'autres familles botaniques, ont ćté contaminćs au moyen d'inoculums de pouvoir infectieux spécifique comparable, mais contenant ou non l'ARN-5, comme cela a été décrit pour les expériences précédentes. Dans tous les cas, nous avons observé une réduction très nette de la gravité des symptômes lorsque l'ARN-5 est présent, en quantité importante (souche $\mathrm{I}_{17} \mathrm{~N}$ ) ou à la dose minimale. En l'absence d'ARN satellite, la croissance de la plante - taille et surface foliaire - est beaucoup plus affectée et les altérations du feuillage sont nettement plus prononcées. La figure 1 présente quelques exemples représentatifs de ces résultats : le tabac, le concombre, le poivron et le basilic, inoculés en présence d'ARN-5 extériorisent, $15 \mathrm{j}$ après l'infection, des symptômes beaucoup plus discrets et leur croissance est moins affectée.

La même expćrience réalisée en additionnant ou non l'ARN-5 de la souche $\mathrm{R}$ à l'ARN viral $\mathrm{I}_{17} \mathrm{~F}$ a conduit à des résultats comparables (résultats non présentés).

\section{DISCUSSION}

Toutes nos souches de VMC pourvues d'un ARN satellite provoquent, à l'exception de la souche $\mathrm{R}$, la nécrose létale de la tomate. L'expression de ce syndrome s'est révélćc particulièrement intéressante en tant qu'indicatrice de la 

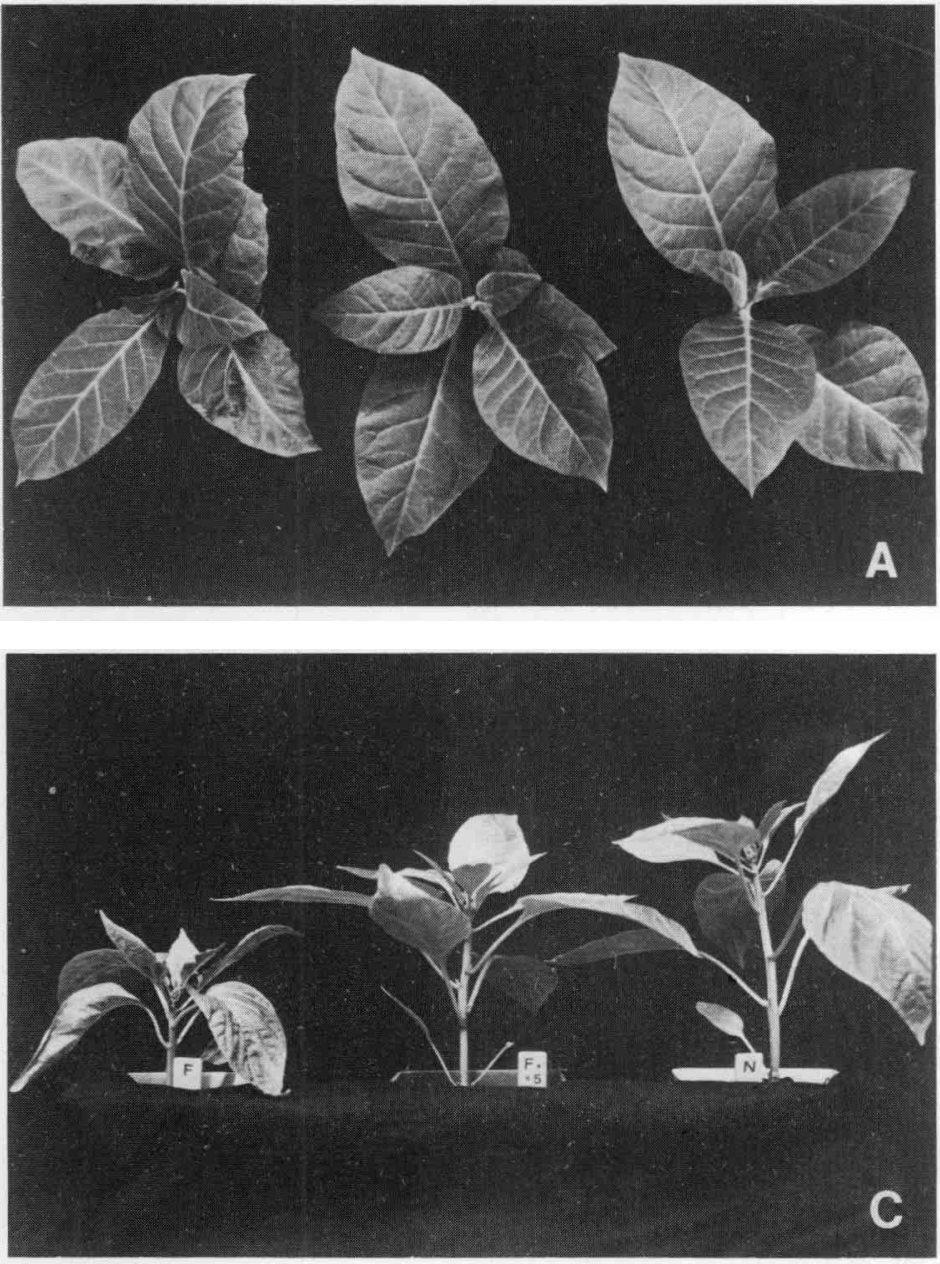

Figure 1

Influence de la présence de l'ARN-5 dans l'inoculum sur l'expression des symptômes développés par 4 hôtes du VMC.

Ces hôtes ont été contaminés par l'ARN de la souche $I_{17} F$ $(20 \mu \mathrm{g} / \mathrm{ml})$ seul (plantes situćes à gauche) ou additionné de l'ARN$5 \mathrm{I}_{17} \mathrm{~N}$ à la dose minimale (plantes situćes au centre) ou par l'ARN de la souche $I_{17} N(40 \mu \mathrm{g} / \mathrm{ml})$ (plantes situćes à droite). Le tabac (A) développe des symptômes de mosaïque très prononcés sculement en l'absence de l'ARN-5. Chez le concombre (B), une nette réduction de la surface des feuilles s'observe également dans ce cas. La moindre altération de la croissance des plantes infectées en présence de l'ARN-5 est ici mise en évidence chez le poivron (C) et le basilic (D).

synthèse de l'ARN satellite. Son intérêt est accru par le fait que le test nécrose constituc une méthode de misc en évidence de l'ARN-5 beaucoup plus facile d'emploi et sensible que l'analyse des composants ARN d'une préparation purifiéc. Nous avons en effet montré que l'ARN-5 est actif à des doses extrêmement réduites. La concentration de $5 \times 10^{-4} \mu \mathrm{g} / \mathrm{ml}$ dans l'inoculum a été définie comme la dose seuil en dessous de laquelle l'efficacité de synthèse de l'ARN satellite n'est plus optimale et, dans le cas des ARN-5 du type D, comme celle qui permet l'induction de nécrose chez la totalité des tomates inoculées. Toutefois, l'ARN satellite, apporté à des concentrations 10000 fois plus faibles, peut toujours être détecté ou induire quelques cas de nécrose. Cette remarquable activité de l'ARN satellite, évoquée par MossoP \& FRANCKI (1979) et WATERWORTH et al. (1979), a été largement utilisće par nous au cours de l'étude de certains aspects de la relation trilatérale virus-ARN satellite-hôte.

Les résultats présentés ici montrent que les ARN-5 de diverses origines que nous avons étudiés ressemblent en de
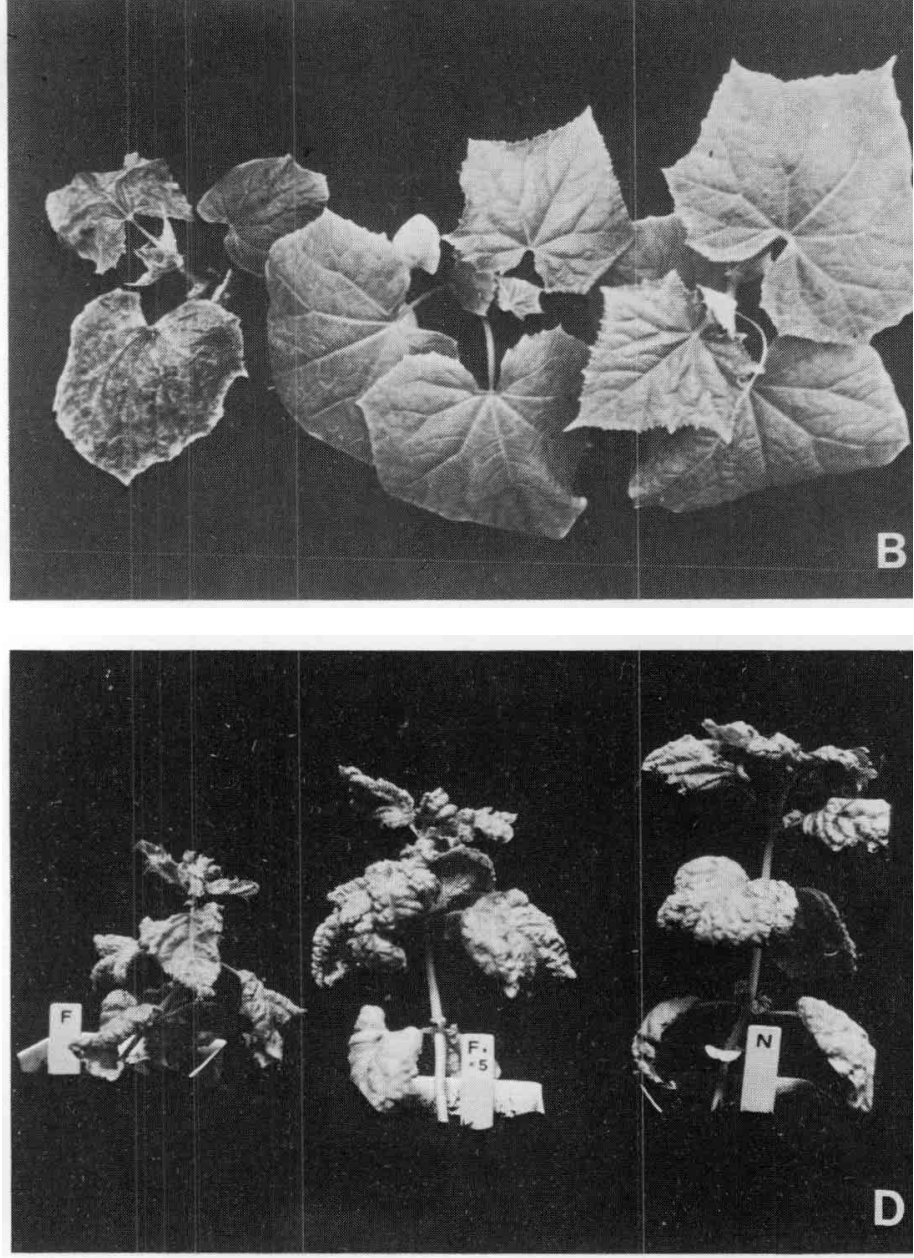

Effect of the presence of RNA-5 in the inoculum on disease symptoms in four species susceptible to $C M V$.

These species were infected with $I_{17} F$ RNA $(20 \mu \mathrm{g} / \mathrm{ml})$ alone (plants on the left) and with $I_{17} \mathrm{~N}$ RNA-5 added at the minimal dose (plants in the middle), or with $\mathrm{I}_{17} \mathrm{~N}$ RNA $(40 \mu \mathrm{g} / \mathrm{ml})$ (plants) on the right). Tobacco (A) develops severe symptoms of mosaic only in the absence of RNA-5. On cucumber (B), a conspicuous reduction of leaf area is also observed in this casc. A lesser changc in plant development is here visible on pepper (C) and basil (D) when RNA-5 is present.

nombreux points aux ARN satellites du VMC décrits par ailleurs :

- Les phénomènes d'interférence existant entre la réplication du virus et celle de l'ARN satellite se traduisent par unc importante réduction de la quantité de virions synthétisés, corollaire de la diminution de synthèse des ARN génomiques (KAPER et al. 1976; GOULD et al., 1978; JACQUEMOND, 1980 ; TAKANAMI, 1981).

- La comparaison de l'aptitude nécrogène d'inoculums correspondant à des combinaisons homologues ou hétérologues de plusieurs souches de virus et d'ARN satellites révèle que toutes les souches dépourvues d'ARN-5 sont capables d'assurer la synthèse de l'ARN satellite quelle que soit son origine. WATERWORTH et al. (1978); MOSSOP \& FRANCKI (1979), TAKANAMI (1981) ont également démontré que le CARNA-5, lc SAT-RNA ou l'ARN-5 isolé d'une souche japonaise du virus, peuvent être assistés par des isolats ne les contenant pas à l'origine. Toutefois, une certaine spécificité apparaît au niveau de l'efficacité de synthèse. 
Celle de l'ARN-5 est en effet dépendante de la nature du génome viral, comme l'ont montré KAPER \& TOUSIGNANT (1977), mais elle est en outre liée à la nature de l'ARN-5 luimême. De plus, la réplication d'un même génome viral est différemment affectée selon l'ARN-5 qui lui est associé.

- Le rôle de l'espèce hôte sur la quantité d'ARN-5 produit est déterminant (KAPER et al., 1976; MOSSOP \& FRANCKI, 1979). Chez tous les hôtes du VMC que nous avons éprouvés, la synthèse de l'ARN-5 même présent à la dose minimale dans l'inoculum, est assurée mais à des taux variables selon l'hôte considéré. Ces variations s'observent plus aisément quand l'ARN-5 est à l'état de traces dans l'inoculum. Les solanacées - et particulièrement le tabac - ainsi que l'épinard sont les plus aptes à assurer la synthèse de l'ARN-5. Des expériences similaires, au cours desquelles des espèces spontanées hôtes du VMC ont été éprouvées dans les mêmes conditions, ont montré que ces plantes sauvages sont toutes sensibles à l'ARN-5 présent à la dose minimale et permettent sa synthèse avec des efficacités variables selon l'espèce infectée (JACQUEMOND, 1980).

Les modifications de l'expression des symptômes chez des hôtes sensibles au virus en présence d'ARN-5 font de l'ARN satellite du VMC un modèle particulicr. En effet, dans tous les autres systèmes virus-satellite étudiés (virus de la nécrose du tabac - TNV - virus des taches annulaires du tabac - TRSV - virus des taches noires de la tomate - TBRV -), l'influence modificatrice du satellite n'a été constatée qu'au niveau du nombre et/ou de l'aspect des lésions locales développées sur les feuilles inoculées des hôtes (KASSANIS \& NIXON, 1961; SCHNEIDER, 1969; MURANT et al., 1973). Les expériences réalisées avec le VMC additionné de concentrations croissantes d'ARN-5 et éprouvé chez un hôte hypersensible, Vigna sinensis, ne nous ont pas permis de conclure à la modification de la taille des lésions locales. En ce qui concerne l'influence de l'ARN-5 sur leur nombre, les résultats sont contradictoires. Dans quelques essais, le nombre de lésions a été réduit proportionnellement à la concentration de l'ARN-5 jusqu'à un certain seuil, dans d'autres essais, par contre, l'apport de l'ARN-5 est resté sans effet (JACQUEMOND, 1980).

Les changements induits par la synthèse de l'ARN satellite dans l'expression des symptômes développés par les espèces sensibles sont, par contre, manifestes. C'est à ce niveau que les principales différences apparaissent entre les divers ARN satellites du VMC. A l'exception de la tomate (et seulement dans le cas d'ARN satellites de type D), tous nos isolats d'ARN-5 provoquent une atténuation des symptômes chez les hôtes inoculés. Avec le tabac en particulier, aucun ARN-5, associé à la souche $\mathrm{I}_{17} \mathrm{~F}$, n'a conduit à la modification de la couleur de la mosaïque comme l'a récemment signalé TAKANAMI (1981). Toutefois, l'ARN-5 qu'il étudie est responsable du développement de nécrose létale chez la tomate comme la majorité de nos isolats. Par contre, l'ARN satellite associć à 2 isolats décrits par
Mossop \& FrANCKI (1979), induit une réduction des symptômes chez la tomate : il serait en cela, comparable à l'ARN-5 de la souche $\mathrm{R}$ que nous possédons. WATERWORTH et al. (1979) ont également décrit une moindre intensité des symptômes développés par le poivron ou le maïs lorsque l'ARN satellite, possédant par ailleurs une activité nécrosante, est présent dans l'inoculum. Ces auteurs ont réalisé leurs expériences avec des extraits bruts de tomates contenant ou non le CARNA-5 et dont les pouvoirs infectieux spécifiques sont, par conséquent, très différents. Les expériences que nous avons réalisées, qui permettent d'éliminer l'effet de la pression de l'inoculum, confirment que, lorsque les plantes sont contaminées à un stade juvénile, la synthèse de l'ARN satellite a pour conséquence une moindre sévérité des symptômes. Cet effet est vraisemblablement lié à la compétition des synthèses du virus et de son ARN satellite. Il n'est pas impossible non plus que l'ARN satellite interfère directement dans la régulation de l'expression des symptômes.

L'origine de l'ARN satellite du VMC n'est pas élucidée. Les isolats étudiés par une même équipe présentent des structures primaires très comparables qui suggèrent une origine commune. La faible spécificité de la relation virusARN satellite, l'intense activité biologique de l'ARN satellite, associées à sa remarquable capacité de «survie » (MOSSOP \& FRANCKI, 1978 ; JACQUEMOND, 1980), peuvent expliquer les risques élevés de contamination du VMC par son ARN satellite. Il est vraisemblable que, dans des conditions naturelles, des souches de VMC dépourvues d'ARN satellite, puissent se trouver, au cours d'infections multiples, associées à un ou des ARN satellites de provenance différente. Au laboratoire, il apparaît préférable de conserver les isolats inducteurs de filiformisme chez la tomate, sous forme de matériel végétal desséché ( $\mathrm{Mc}$ KINNEY, 1974) après leur multiplication chez un hôte peu favorable à la synthèse de l'ARN satellite (Cucurbitacée). Il nous semble peu probable que l'ARN-5 puisse être naturellement induit chez le tabac durant la réplication virale ainsi que WaterwORTH et al. (1977) en font l'hypothèse. En effet, plusieurs isolats, dont les souches $I_{17} F$ et $R$, s'avèrent toujours incapables de provoquer la nécrose de la tomate après avoir été préalablement multipliés chez le tabac. La synthèse de l'ARN satellite chez cet hôte infecté par un isolat ne le contenant apparemment pas nous paraît plus vraisemblablement liée à l'extrême sensibilité du tabac à la présence d'ARN satellite en quantité infinitésimale dans l'inoculum.

\section{REMERCIEMENTS}

Les auteurs remercient Hervé LoT pour les remarques constructives dont il leur a fait part au cours de la réalisation de ce travail. Les photographies sont dues à Jacque RovGIER.

Reçu le $1^{\text {er }}$ juin 1981. Accepté le 29 septembre 1981.

\section{RÉFÉRENCES BIBLIOGRAPHIQUES}

Diaz-Ruiz J. R., Kaper J. M., 1977. Cucumber mosaic virus-associated RNA-5. III. Little nucleotide sequence homology between CARNA-5 and helper RNA. Virology, 80, 204-213.

Gould A. R., Palukaitis P., Symons R. H., Mossop D. W., 1978. Characterization of a satellite RNA associated with cucumber mosaic virus. Virology, 84, 443-445.

Jacquemond M., 1980. Propriétés et fonctions biologiques du satellite du virus de la mosaïque du concombre. La nécrose de la tomate. Thèse du Docteur-ingénieur, Université d'Aix-Marseille, $167 \mathrm{pp}$.

Jacquemond M., Lot H., 1981. L'ARN satellite du virus de la mosaïque du concombre. I. Comparaison de l'aptitude à induire la nécrose de la tomate d'ARN satellites isolés de plusicurs souches du virus. Agronomie, 1, (10) (sous presse).

Kaper J. M., Tousignant M. E., 1977. Cucumber mosaic virus- 
associated RNA-5. I. Role of host plants and helper strains in determining amount associated with virions. Virology, 80, 186-195.

Kaper J. M., Tousignant M. E., 1978. Cucumber mosaic virusassociated RNA-5. V. Extensive nucleotide sequence homology among CARNA-5 preparations of different CMV strains. Virology, 85, 323-327.

Kaper J. M., Tousignant M. E., Lot H., 1976. A low molccular weight replicating RNA associated with a divided genome plant virus: defective or satellite RNA? Biochem. Biophys. Res. Comm., 72, (4), 1237-1243.

Kaper J. M., Waterworth H. E., 1977. Cucumber mosaic virusassociated RNA-5 causal agent for tomato necrosis. Science, 196, 429-431.

Kassanis B., Nixon H. L., 1961. Activation of one tobacco necrosis virus by another. J. Gen. Microbiol., 25, 459-471.

Lot H., Jonard G., Richards K. E., 1977. Cucumber mosaic virus RNA-5. Partial characterization and evidence for no large sequence homologies with genomic RNAs. Febs Letters., 80 (2), 395-399.

Mc Kinney H. H., 1947. Stability of labile viruses in dessicated tissue. Phytopathology, 37 (2), 134-142.

Mossop D. W., Francki R. I. B., 1978. Survival of a satellite RNa in vivo and its dependence on cucumber mosaic virus for replication. Virology, 86, 562-566.
Mossop D. W., Francki R. I. B., 1979. Comparative studies on two satellites RNAs of cucumber mosaic virus. Virology, 95, 395-404. Murant A. F., Mayo M. A., Harrison B. d., Goold R. A., 1973. Evidence for two functional RNAs species and a "satellite " RNA in tomato black ring virus. J. Gen. Virol., 19, 275-278.

Richards K. E., Jonard G., Jacquemond M., Lot H., 1978. Nucleotide sequence of cucumber mosaic virus-associated RNA-5. Viro$\log y, 89,395-408$.

Schneider I. R., 1969. Satellite-like particle of tobacco ringspot virus that resembles tobacco ringspot virus. Science, 166, 16271629.

Takanami Y., 1981. A striking change in symptoms on cucumber mosaic virus-infected tobacco plants induced by a satellitc RNA. Virology, 109, 120-126.

Waterworth H. E., Kaper J. M., Tousignant M. E., 1979. CARNA-5, the small cucumber mosaic virus-dependent replicating RNA, regulates disease expression. Science, 204, 845-847.

Waterworth H. E., Tousignant M. E., Kaper J. M., 1978. A lethal disease of tomato experimentally induced by RNA- 5 associated with cucumber mosaic virus isolated from Commelina from El Salvador. Phytopathology, 68, 561-566. 